\title{
Editorial: In Silico Characterization of Inhibitors Versus Resistant Plasmodium Falciparum Entangled in Malaria Prevention
}

\section{Sanjay Mishra*}

School of Biotechnology, IFTM University, India

*Corresponding author: Sanjay Mishra, School of Biotechnology, IFTM University, Delhi Road (NH 24), Moradabad- 244102, Uttar Pradesh, India, Email: sanjaymishra@iftmuniversity. ac.in; sanjaymishra66@gmail.com

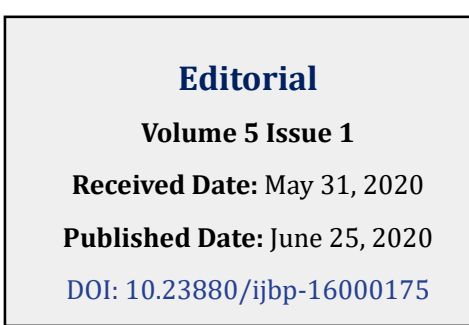

\section{Editorial}

Malaria is an ancient disease, having been known to be one of the fatal, life-threatening communicable diseases, caused by a single celled parasite, Plasmodium falciparum $(P$. falciparum). Drug resistance against malaria parasite refers to almost every known anti- malarial agent, underscoring the case by which parasite population can adapt and survive. Resistance of the malaria parasite, mainly to chloroquine and to the antifolates, will continue to make progress in rolling back malaria a formidable challenge for the foreseeable future. Antifolates antimalarial drugs interfere with folate metabolism, essential to malaria parasite survival. Unfortunately, the antifolates have proven susceptible to resistance in the malaria parasite. Resistance is caused by point mutations in dihydrofolate reductase, the key enzyme in the folate biosynthesis, targeted by the antifolates. Disruption of folate synthesis by DHFR inhibitor leads to decreased levels of fully reduced tetrahydrofolate, resulting in decreased conversion of glycine to serine, reduced methionine synthesis, and lower thymidylate levels with a subsequent arrest of DNA replication.

The malaria parasite also relies exclusively on de novo pyrimidine biosynthesis to supply precursors for DNA and RNA biosynthesis. In contrast, the human host cells contain the enzymatic machinery for both de novo pyrimidine biosynthesis and for salvage of preformed pyrimidine bases and nucleosides. The lack of a redundant mechanism to acquire pyrimidines in malaria has raised interest in this pathway as a potential source for new therapeutic targets. Dihydroorotate dehydrogenase (DHODH) is a flavindependent mitochondrial enzyme that catalyzes fourth reaction of pyrimidine de-novo synthesis. Pyrimidine bases are essential for cellular metabolism and cell growth, glycoproteins and phospholipids biosynthesis. The significance of pyrimidines biosynthesis in DNA and RNA makes them ideal targets for pharmacological intervention.
Inhibitors of DHODH have proven efficacy for the treatment of malaria, autoimmune diseases, cancer, rheumatoid arthritis and psoriasis.

The Plasmodium falciparum dihydrofolate reductase (DHFR) and dihydroorotate dehydrogenase (DHODH) are drug targeting proteins for the drug discovery fighting with the malaria.

Computer-aided drug designing (CADD) tools are playing a major role in drug discovery processes and are being used successfully for the development of new drug targets or therapeutically important small molecules in medical field. In a drug discovery process, CADD is usually used for filtering large chemical compound, for optimization of lead compounds in terms of increasing its affinity or for optimization of drug metabolism and pharmacokinetics properties together with absorption, distribution, metabolism, excretion, and the potential for toxicity properties for designing novel compounds. Analogues of Pyrimidine and benzamide have been computionally designed followed by docking with $P$. falciparum dihydrofolate reductase (PfDHFR) and dihydroorotate dehydrogenase (PfDHODH) proteins using AutoDock, respectively [1,2]. Interaction between the PfDHFR and PfDHODH proteins with Pyrimidine and benzamide derivatives were analysed through Python Molecular Viewer software, respectively.

Proguanil is a drug for malaria, creating hindrance in reproduction of malaria parasite. Proguanil inhibits the enzyme DHFR from P. falciparum and P. vivax. Hex software has been used for conformational analysis and geometrical optimization of Proguanil [3].

The Docking and binding affinity analysis revealed that most of the pathogen proteins involved in the infection 


\section{International Journal of Biochemistry \& Physiology}

process are structural and assembly proteins such as tubulin, actin, and histone or nuclear assembly proteins [4]. Hence, the pathogen caused infection by targeting nuclear assembly proteins, inhibiting the proper function of host cell [4], thus, $\alpha$-tubulin from pathogen to be used for improvement of antimalarial agent.

Optimal interactions and the best autodock score were used as criteria to interpret the most favoured conformation among thirty conformations, generated by AutoDock program. The docking energy results of twenty seven compounds with PfDHFR were summarized and the compound having CID 10476801 was found to have least docking energy with PfDHFR in comparison to other docked compounds [5]. The analysis of docked compound with Python molecular viewer revealed the hydrogen bond formation between active site, ASP 54 residues of the Pf DHFR inhibitor as well as the compound CID10476801(benzyl 3-(2,6-diamino-5-(3chlorophenyl)pyrimidin-4-yl)propyl carbonate) [5]. Similarly the docking of twenty five screened benzamide analogues with PfDHODH predicted the compound CID 867491((N-(4bromo-3-methylphenyl)-2- methyl-3-nitrobenzamide) to have lowest docking energy and to having hydrogen bond with HIS185 amino acid residue of Pf DHODH enzyme [6].

\section{Conclusion and Future Perspectives}

The PfDHFR is a drug targeting protein for the drug discovery fighting with the malaria. Docking study predicted that compound CID 10476801 and CID 867491 have lowest docking energy ( $-11.48 \mathrm{kcal} / \mathrm{mol}$ and $-4.82 \mathrm{Kcal} / \mathrm{mol}))$ with PfDHFR and PfDHODH, respectively and their interaction are stabilized by hydrogen bonding, playing an important role for the structure and function of potent inhibitor molecules. Computational methods assist the drug designing by screening the huge amount of ligand from large chemical database and predicting the interaction and stability of docked complex with target protein, which reduces the cost and time, spent by using traditional methods, providing new insights into validating the identified molecule in wet lab leading towards drugs to be successfully brought into practice in view as an active antimalarial drug molecule.

\section{References}

1. Phillips MA, Rathod PK (2010) Plasmodium dihydroorotate dehydrogenase: a promising target for novel anti-malarial chemotherapy. Infectious disorders drug targets 10(3): 226-239.

2. Vyas VK, Qureshi G, Ghate M, Patel H, Dalai S (2016) Identification of novel PfDHODH inhibitors as antimalarial agents via pharmacophore-based virtual screening followed by molecular docking and in vivo antimalarial activity. SAR QSAR Environ Res 27(6): 427-440.

3. Prakash N, Patel S, Faldu NJ, Ranjan R, Sudheer DVN (2010) Molecular Docking Studies of Antimalarial Drugs for Malaria. J Comput Sci Syst Biol 3(3): 070-073.

4. Monika Samant,1 Nidhi Chadha, Anjani K. Tiwari, Yasha Hasija (2016) In Silico Designing and Analysis of Inhibitors against Target Protein Identified through Host-Pathogen Protein Interactions in Malaria. Int J Med Chem 2016: 2741038.

5. Singh IV, Mishra S (2018) Molecular Docking Analysis of Pyrimethamine Derivatives with Plasmodium falciparum Dihydrofolate reductase. Bioinformation 14(5): 232235.

6. Vikram Singh I, Mishra S (2019) Molecular Docking Studies of Benzamide Derivatives for PfDHODH Inhibitor as Potent Antimalarial Agent. American Journal of Biochemistry and Molecular Biology 9: 1-6. 